Case Report

\title{
Schwannoma in Lower Lip - A case Report
}

\author{
Joshi $\mathbf{S}^{1}$, Acharya $\mathbf{S}^{2}$, Chaulagain $\mathbf{R}^{3}$ \\ ${ }^{1}$ Lecturer, Department of Oral and Maxillofacial Surgery, KISTMCTH \\ ${ }^{2}$ Associate Professor, Department of Oral and Maxillofacial Surgery, KISTMCTH \\ ${ }^{3}$ Dental Surgeon, Ph.D Researcher, Department of Oral and Maxillofacial Surgery, KISTMCTH \\ KISTMCTH- Kist Medical College and Teaching Hospital
}

Correspondence : Dr . Sandhya joshi Email: sandhyajo@hotmail.com

\begin{abstract}
Introduction: Schwannoma is a benign tumor arising from and consisting solely of Schwann cells. These are usually single and well encapsulated lesion. The most common intraoral site is tongue followed by palate, buccal mucosa, lip, gingiva and jaws. However, here we report a case of schwannoma of lower labial mucosa which was asymptomatic for 8 years. The histopathological examination revealed distinct Antoni A and Antoni B cells confirming the diagnosis of schwannoma. Nevertheless the rare entity such as schwannoma of labial mucosa should be kept in differential diagnosis for any nodule or mass in the labial mucosa of the oral cavity.
\end{abstract}

Keywords: schwannoma

\section{Introduction}

The schwannoma (neurilemmoma) is a neurogenic benign tumor that arises from the Schwann cells of the nerve sheath. There is a large variety of terms for schwannomas in the literature, but only three are still in current use: neurinoma, neurilemmoma and schwannoma. Schwannoma is a relatively uncommon benign, slow-growing, encapsulated tumor that typically arises in association with a nerve trunk and as it grows it pushes the nerve aside ${ }^{1}$. The cause of the tumor is unknown. However, some etiological factors are conjectured, such as spontaneous growth, external injury, chronic irritation, or exposure to radiation ${ }^{2}$. Schwannomas of the oral cavity are one of the rare oral peripheral nerve tumors. About $25 \%$ of the schwannomas appear in the head and neck region, but only $1 \%$ are located in the oral cavity, showing a predilection for the tongue, followed by the palate, buccal mucosa, lip, and gingiva ${ }^{2,3}$.

The schwannomas in oral soft tissue appear as a smooth submucosal swelling, thus resembling other lesions like mucocele, fibro epithelial polyp, fibroma, lipoma and benign salivary gland tumors. The histological differential diagnosis will be with other neural origin lesions, which could be neurofibroma and neuroma, muscular or fibroblastic origin tumor ${ }^{3}$. Although this tumor can appear at any age, it is more commonly seen in the fourth decade ${ }^{4}$.

Here we report a case of schwannoma which had clinical diagnosis of lipoma, later on through histopathological examination the final diagnosis of schwannoma was made.

\section{Case Report}

A 18 - year old male presented to our Department of Oral and Maxillofacial Surgery with a complaint of swelling in the lower lip since 8 years. He noticed it as a small nodule which was painless and without any discharge. However gradually over the years the swelling has been increasing in size and has reached to the present size. On examination a firm smooth mass was noted on the lower labial mucosa on the left side [Fig 1]. The swelling measured approximately $1.5 \mathrm{~cm}$ in greatest dimension. The overlying mucosa was normal in color, fluctuation was negative and slip sign was positive indicating its solidity in nature. The clinical diagnosis of lipoma was made and therefore excision of the mass was carried out after hematological investigations [Figure 2]. 
The excised mass was sent for histopathological examination which revealed encapsulated lesion with Antoni A and Antoni B areas. Antoni A area comprised cellular areas. The cells were arranged in bundles, fascicles and whorled pattern; and had spindle nuclei and indistinct cytoplasm. There were few cells that show enlarged, hyperchromatic nuclei but mitoses was not seen. Verocay bodies, Antoni $\mathrm{B}$ area showing loose edematous, myxoid and hyalinised areas were seen [Figure 3]

The post-operative period was uneventful and is under follow up.

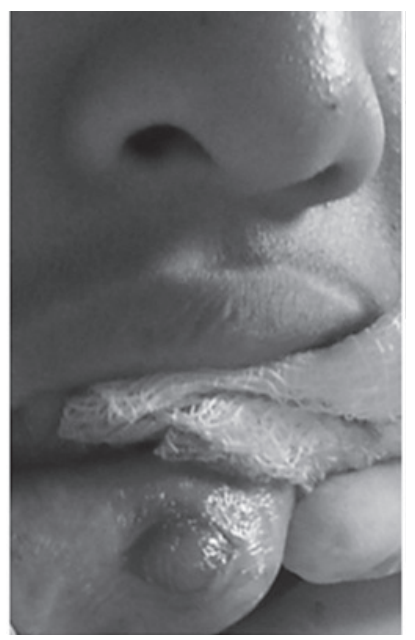

Figure 1. Picture showing swelling on the labial mucosa

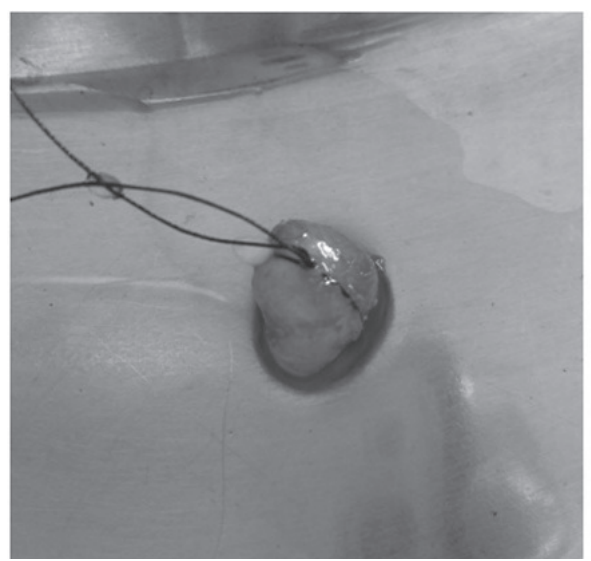

Figure 2. Picture showing excised mass

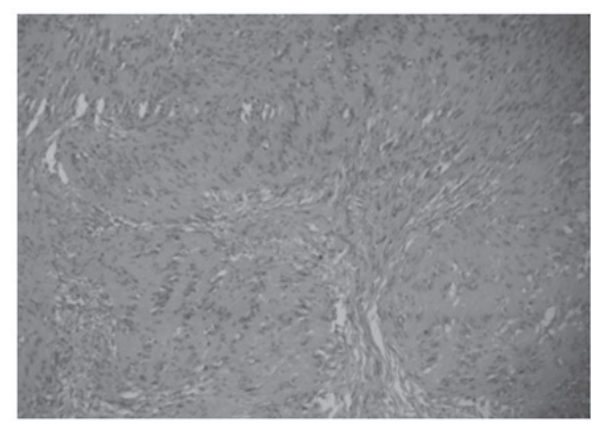

Figure 3. Antoni A and Verocay bodies seen (H\&E staining)

\section{Discussion}

Schwannoma is a benign, normally asymptomatic, solitary, encapsulated neoplasm originated from the Schwann cells of the nerve sheath, and occurs along the course of a somatic, cranial, or sympathetic nerve ${ }^{4}$.

It is believed to originate from proliferation of schwann cells in the perineurium causing displacement and compression of adjacent nerve.It does not arise from cranial nerve I and II because they lack Schwann cells ${ }^{3}$. Schwannoma of the lip is a rarely reported clinical entity ${ }^{7}$.

Microscopically, these tumors have two distinct architectural patterns of growth labeled as Antoni A and Antoni B. The Antoni A areas are characterized by the compact arrangement of the neoplastic cells with frequent nuclear palisading and the presence of Verocay bodies, whereas Antoni B areas show decreased cellularity and have an extracellular matrix with a loose aspect and delicate collagen fibers and scarce mononuclear inflammatory cells ${ }^{6}$.

Schwannomas generally cannot be diagnosed preoperatively, unless the patient has a personal or family history of other neural lesions. These lesions can be part of the von Recklinghausen's disease (neurofibromatosis I), which is characterized by spots of increased pigmentation of the skin (café au lait spots) combined with peripheral nerve tumors and a variety of other abnormalities of the skin, nervous system, bones, endocrine organs, and blood vessels $^{6}$.

However microscopically, both schwannoma and the neurofibroma contain elongated cells with irregular nuclei lying between bundles of collagen fibers. They differ histologically and histogenetically, as the schwannoma is derived from the Schwann cells and the neurofibroma from the fibroblasts of the perineurium. Neurofibroma is 
unencapsulated, consisting of a mixture of Schwann cells, perineurial cells, and endoneurial fibroblasts.

In our case, histologically it showed encapsulated lesion with Antoni A and Antoni B areas. Antoni A area comprise cellular areas with the cells arranged in bundles, fascicles and whorled pattern. Antoni B area showed loose edematous, myxoid and hyalinised areas. In the present case, the histopathologic analysis revealed a majority of Antoni A pattern for the whole specimen and thick walled blood vessels were also noted in the section.

On Immunohistochemistry, S-100 protein shows strong positivity for neurilemmomas, reflecting their propensity of Schwann cells. They also show positivity for vimentin, Leu-7 antigen and glial fibrillary acidic protein ${ }^{6}$.

The treatment of schwannoma is complete excision of the lesion and the prognosis is favorable. No malignant transformation has been reported, although local recurrence is possible and has been associated with incomplete resection of the tumor ${ }^{7}$.

\section{Conclusion}

Intraoral Schwannoma although is a rare entity should be kept in a differential diagnosis for any asymptomatic well circumscribed nodule or mass. The entity has a remarkable prognosis and treatment is almost curative with complete excision.

\section{References}

1. Lambade PN, Palve D, Lambade D. Schwannoma of the cheek: clinical case and literature review J Maxillofac Oral Surg 2015;14(2):327-31.

2. Hwang K, Kim SG, Ahn SI, Lee SI. Neurilemmoma of the tongue. Journal of Craniofacial Surgery. $2005 ; 16(5): 859-861$

3. B. Shilpa. Case report ancient schwannoma-a rare case. Ethiop J Health Sci.2012; 22(2):215-218.

4. Da silva LF, Duarte BG, Boica BA, Rocha- Junior HV, Pereira- Stabile CL. Intraoral Schwannoma: a case report Oral Maxillofac Surg. 2013 Dec;17(4):319-2

5. Martins MD, Anunciato de Jesus L, Fernandes KP, Bussadori SK, Taghloubi SA, Martins MA. Intra-oral schwannoma: case report and literature review. Indian journal of dental research: 2009; 20(1):121-5
6. Sahoo PK, Mandal PK, Ghosh S Schwannoma of the hard palate. Natl J Maxillofac Surg. 2014; 5(1): 39-41.

7. Cardosa CL, Tolentino Ede S, CapelozzaAL, Consolaro A. Schwannoma in the lower lip mucosa: unexpected diagnosis, Quintessence Int 2010;41(9):769-71

8. Weiss SW, Goldblum JR (2001) Benign tumors of peripheral nerves. In: Soft tissue tumors, 4th edn. Mosby-Year Book, St. Louis, pp. 1111-1200 